\title{
ON THE FOURTH COEFFICIENT OF THE INVERSE OF A STARLIKE FUNCTION OF POSITIVE ORDER
}

\author{
TOSHIYUKI SUGAWA AND LI-MEI WANG
}

\begin{abstract}
We consider the inverse function $z=g(w)$ of a (normalized) starlike function $w=f(z)$ of order $\alpha$ on the unit disk of the complex plane with $0<\alpha<1$. Krzyż, Libera and Złotkiewicz obtained sharp estimates of the second and the third coefficients of $g(w)$ in their 1979 paper. Prokhorov and Szynal gave sharp estimates of the fourth coefficient of $g(w)$ as a consequence of the solution to an extremal problem in 1981. We give a straightforward proof of the estimate of the fourth coefficient of $g(w)$ together with explicit forms of the extremal functions.
\end{abstract}

\section{INTRODUCTION}

We denote by $\mathcal{A}$ the set of analytic functions $f(z)$ on the unit disk $\mathbb{D}=\{z \in \mathbb{C}:|z|<1\}$ normalized by $f(0)=f^{\prime}(0)-1=0$. Let $\mathcal{S}$ denote the subset of $\mathcal{A}$ consisting of univalent ones. Each function $f \in \mathcal{S}$ is expanded in the form

$$
f(z)=z+\sum_{n=2}^{\infty} a_{n} z^{n} .
$$

The Bieberbach conjecture asserts that $\left|a_{n}\right| \leq n$ for $n \geq 2$ and it is finally proved completely by de Branges in 1984 (see, for instance, [4]). We note that equality holds for the Koebe function $K(z)=z /(1-z)^{2}=z+2 z^{2}+3 z^{3}+\cdots$. The Koebe one-quarter theorem implies that $f(\mathbb{D})$ contains the disk $|w|<1 / 4$ so that the inverse function $z=g(w)$ of a univalent function $w=f(z)$ in $\mathcal{S}$ is analytic on $|w|<1 / 4$. We expand $g=f^{-1}$ in the form

$$
g(w)=w+\sum_{n=2}^{\infty} b_{n} w^{n} .
$$

Löwner [10] showed the sharp inequalities

$$
\left|b_{n}\right| \leq \frac{(2 n) !}{n !(n+1) !}=\frac{1}{n+1}\left(\begin{array}{c}
2 n \\
n
\end{array}\right), \quad n=2,3, \ldots
$$

(see also $[4, \S 7.9])$. We note that equalities hold for $K^{-1}(w)=[1-2 w-\sqrt{1-4 w}] /(2 w)$. A function $f \in \mathcal{A}$ is called starlike of order $\alpha$ if

$$
\operatorname{Re}\left(\frac{z f^{\prime}(z)}{f(z)}\right)>\alpha, \quad z \in \mathbb{D}
$$

2010 Mathematics Subject Classification. Primary 30C45; Secondary 30C50.

Key words and phrases. starlike function of order $\alpha$, inverse function, coefficient estimates.

The first author is supported in part by JSPS KAKENHI Grant Number JP17H02847. The second author is supported by "the Fundamental Research Funds for the Central Universities" in UIBE (No. 18YB02) and National Natural Science Foundation of China (No. 11901086). 
for a constant $\alpha \in[0,1)$. We denote by $\mathcal{S}^{*}(\alpha)$ the set of those functions $f$. A function in $\mathcal{S}^{*}(0)=: \mathcal{S}^{*}$ is simply called starlike and known to map $\mathbb{D}$ univalently onto a starlike domain in $\mathbb{C}$ with respect to the origin. Hence $\mathcal{S}^{*}(\alpha)\left(\subset \mathcal{S}^{*}\right)$ is contained in $\mathcal{S}$ for $0 \leq$ $\alpha<1$. For a function $f$ in $\mathcal{S}^{*}(\alpha)$, Robertson [12] proved the sharp inequality

$$
\left|a_{n}\right| \leq \frac{\Gamma(2-2 \alpha+n-1)}{\Gamma(2-2 \alpha) \Gamma(n)}=\frac{(2-2 \alpha)_{n-1}}{(n-1) !},
$$

for each $n \geq 2$. Here, $(a)_{n}$ is the Pochhammer symbol and means $a(a+1) \cdots(a+n-1)$ for $n \geq 1$ and $(a)_{0}=1$. Equalities hold above for the function

$$
K_{\alpha}(z)=\frac{z}{(1-z)^{2(1-\alpha)}}=\sum_{n=1}^{\infty} \frac{(2-2 \alpha)_{n-1}}{(n-1) !} z^{n} .
$$

It is also known that $f(\mathbb{D})$ contains the disk $|w|<2^{-2(1-\alpha)}$ for every $f \in \mathcal{S}^{*}(\alpha)$. However, sharp inequalities are less known for the inverse functions of starlike functions of order $\alpha>0$. Krzyż, Libera and Złotkiewicz [6] gave the sharp estimates

$$
\left|b_{2}\right| \leq 2(1-\alpha)
$$

and

$$
\left|b_{3}\right| \leq \begin{cases}(1-\alpha)(5-6 \alpha) & \text { for } 0 \leq \alpha \leq \frac{2}{3} \\ 1-\alpha & \text { for } \frac{2}{3} \leq \alpha<1 .\end{cases}
$$

Equality is attained by $g=K_{\alpha}^{-1}$ for $\left|b_{2}\right|$ and for $\left|b_{3}\right|$ with $0 \leq \alpha \leq \frac{2}{3}$ and by $g=K_{\alpha, 2}^{-1}$ for $\left|b_{2}\right|$ with $\frac{2}{3} \leq \alpha<1$, where

$$
K_{\alpha, n}(z)=\left\{K_{\alpha}\left(z^{n}\right)\right\}^{1 / n}=\frac{z}{\left(1-z^{n}\right)^{2(1-\alpha) / n}}, \quad 0 \leq \alpha<1, n=1,2,3, \ldots
$$

Campschroer [2, Theorem 2.IX] proved the following theorem, except for the explicit expression of the bound in the first case and for the second case, both of which were shown by Kapoor and Mishra [5].

Theorem A. Let $f^{-1}(w)=w+b_{2} w^{2}+b_{3} w^{3}+\cdots$ for an $f \in \mathcal{S}^{*}(\alpha)$. Then

$$
\left|b_{n}\right| \leq \begin{cases}\frac{(n-2 n \alpha+2)_{n-1}}{n !} & \text { for } 0 \leq \alpha \leq \frac{2}{n} \\ \frac{(n-2 n \alpha+k+1)_{n-k}}{n(n-1)(n-k-1) !} & \text { for } \frac{k}{n} \leq \alpha<\frac{k+1}{n}, k=2, \ldots, n-2, \\ \frac{2(1-\alpha)}{n-1}, & \text { for } \frac{n-1}{n} \leq \alpha<1 .\end{cases}
$$

Moreover, equality holds when $f=K_{\alpha}$ for the first case, and equality holds when $f=$ $K_{\alpha, n-1}$ for the last case.

We remark that the above result was extended to the so-called Janowski starlike functions by Ali and Vasudevarao [1].

Though the sharp bound of $\left|b_{n}\right|$ is not known for $n \geq 5$ and $\frac{2}{n}<\alpha<\frac{n-1}{n}$, Prokhorov and Szynal [11] obtained the sharp bounds of $\left|b_{4}\right|$ for $(\alpha, \beta)$-convex functions, which contains the case of starlike functions of order $\alpha$. Indeed, for given real numbers $\mu$ and $\nu$, they solved the extremal problem of finding the maximum $\left|e_{3}+\mu e_{1} e_{2}+\nu e_{1}^{3}\right|$ over all the 
analytic functions $\omega(z)=e_{1} z+e_{2} z^{2}+e_{3} z^{3}+\cdots$ on $\mathbb{D}$ with $|\omega(z)| \leq|z|$, and then they derived their main results from the solution to this extremal problem (see also [2, Lemma 2.VI]). They also provided the information of extremal functions in terms of the function $\omega$ determined by the relation $z f^{\prime}(z) / f(z)=(1+(1-2 \alpha) \omega(z)) /(1-\omega(z))$. Therefore, the expression of the extremal functions is not very explicit. We will give a more detailed proof of it with explicit forms of extremal functions as follows. (See also [2, Theorem 2.VIII].)

Theorem 1.1 ( $c f$. Prokhorov-Szynal [11, Theorem 2"]). Let $f^{-1}(w)=w+b_{2} w^{2}+b_{3} w^{3}+$ $b_{4} w^{4}+\cdots$ for a function $f \in \mathcal{S}^{*}(\alpha)$ with $0 \leq \alpha<1$. Then the following sharp inequality holds:

$$
\left|b_{4}\right| \leq \begin{cases}\frac{2}{3}(1-\alpha)(3-4 \alpha)(7-8 \alpha) & \text { if } 0 \leq \alpha \leq \alpha_{1}=\frac{3}{5} \\ \frac{4(2-3 \alpha)^{3 / 2}(3-4 \alpha)(7-8 \alpha)}{3 \sqrt{5-8 \alpha}} & \text { if } \alpha_{1} \leq \alpha \leq \alpha_{2}=\frac{35-\sqrt{33}}{48} \approx 0.609488 \\ \frac{2}{3}\left(\frac{11-12 \alpha}{6}\right)^{3 / 2} & \text { if } \alpha_{2} \leq \alpha \leq \alpha_{3}, \\ \frac{2}{3}(1-\alpha) & \text { if } \alpha_{3} \leq \alpha<1,\end{cases}
$$

where

$$
\alpha_{3}=\frac{1}{24}(21-\sqrt[3]{13+2 \sqrt{11}}-\sqrt[3]{13-2 \sqrt{11}}) \approx 0.685369
$$

If $0 \leq \alpha \leq \alpha_{1}$, then equality holds for $f=K_{\alpha}$.

If $\alpha_{1} \leq \alpha \leq \alpha_{2}$, then equality holds for

$$
f(z)=\frac{z}{(1-z)^{2(1-\alpha) \lambda}\left(1-e^{i \theta_{0}} z\right)^{2(1-\alpha)(1-\lambda)}},
$$

where

$$
\begin{gathered}
\theta_{0}=\arccos \left[\frac{4\left(72 \alpha^{2}-103 \alpha+36\right)}{5-8 \alpha}\right] \\
\lambda=\frac{(5 \alpha-3)(7-8 \alpha)}{2(1-\alpha)^{2}(72 \alpha-43)-(1-\alpha) \sqrt{(3 \alpha-2)(72 \alpha-43)\left(24 \alpha^{2}-33 \alpha+11\right)}} . \\
\text { If } \alpha_{2} \leq \alpha \leq \alpha_{3} \text {, then equality holds for }
\end{gathered}
$$

$$
f(z)=\frac{z}{(1+z)^{(1-\alpha)(1-s)}(1-z)^{(1-\alpha)(1+s)}}, \text { where } s=\sqrt{\frac{11-12 \alpha}{4 \sqrt{6}(1-\alpha)}} .
$$

If $\alpha_{3} \leq \alpha<1$, then equality holds for $f=K_{\alpha, 3}$.

Let $B(\alpha)$ be the sharp bound for $\left|b_{4}\right|$ given in the above theorem. The graph of the function $B(\alpha)$ in the range $1 / 2 \leq \alpha<1$ is exhibited in Figure 1 .

The organization of the present note is as follows. In the next section, a few preliminary results are given. Some of them may be useful in different situations. Section 3 will be devoted to the proof of the main theorem except for the technical Lemma 3.1, which will be shown in Section 4. 


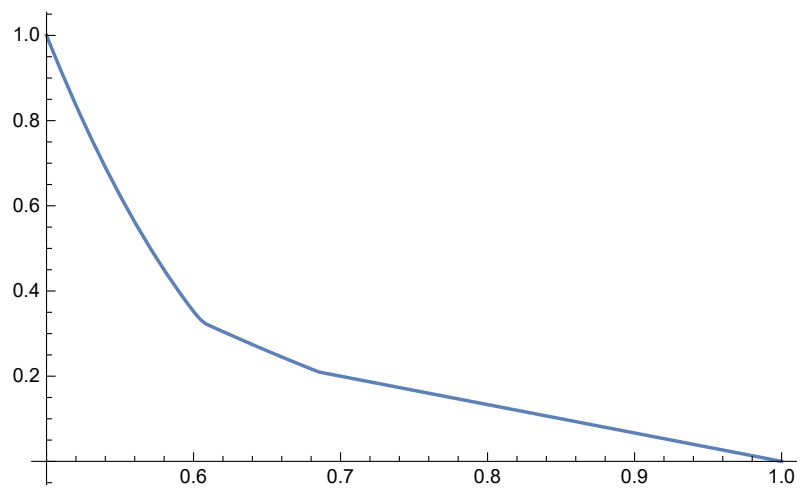

Figure 1 . The graph of the bound $B(\alpha)$ for $\left|b_{4}\right|$ in the range $1 / 2 \leq \alpha<1$

As the reader will observe, our proof is quite involved with heavy computations. It is quite surprising that Prokhorov and Szynal were able to show it (even in a much general form) as early as in 1981. Finally, the authors would like to thank Professor Ponnusamy for conveying the paper [11] to their attention. Before that, the authors prepared the manuscript, being unaware of [11]. The authors believe that the present paper has a merit in the sense that it may give new techniques to the reader to attack similar problems.

\section{Preliminaries}

Recall that an analytic function $p$ on $\mathbb{D}$ with $p(0)=1$ and $\operatorname{Re} p>0$ is called a Carathéodory function. We will denote by $\mathcal{P}$ the set of Carathéodory functions. Therefore, we can say that a function $f \in \mathcal{A}$ is starlike of order $\alpha$ if and only if $p(z)=\left[z f^{\prime}(z) / f(z)-\right.$ $\alpha] /(1-\alpha)$ belongs to $\mathcal{P}$. In this case, we can write

$$
\frac{z f^{\prime}(z)}{f(z)}=\alpha+(1-\alpha) p(z)
$$

It is therefore fundamental to investigate the coefficients of Carathéodory functions in order to study starlike functions of order $\alpha$. We will introduce several necessary results in this section. The next result is essentially due to Libera and Złotkiewicz [9] (the present version is found in [8]).

Lemma 2.1. Let $p(z)=1+2 c_{1} z+2 c_{2} z^{2}+2 c_{3} z^{3}+\cdots$ be a Carathéodory function. Then

$$
\begin{aligned}
& c_{1}=u, \\
& c_{2}=u^{2}+v\left(1-|u|^{2}\right), \\
& c_{3}=u^{3}+\left(1-|u|^{2}\right) v(2 u-\bar{u} v)+\left(1-|u|^{2}\right)\left(1-|v|^{2}\right) w
\end{aligned}
$$

for some $u, v, w \in \overline{\mathbb{D}}$. Conversely, for complex numbers $c_{1}, c_{2}, c_{3}$ expressed as above, there is a Carathéodory function $p$ satisfying $p(z)=1+2 c_{1} z+2 c_{2} z^{2}+2 c_{3} z^{3}+O\left(z^{4}\right)$.

We will also make use of the next result derived from Carathéodory-Toeplitz theorem (see [7, Lemma 2.6]). 
Lemma 2.2. Let $p(z)=1+2 c_{1} z+2 c_{2} z^{2}+\cdots$ be a Carathéodory function with $c_{1}=c \in$ $[0,1)$. If $c_{2}=c^{2}+v\left(1-c^{2}\right)$ for some $v \in \mathbb{C}$ with $|v|=1$, then

$$
p(z)=\lambda_{1} \frac{1+\varepsilon_{1} z}{1-\varepsilon_{1} z}+\lambda_{2} \frac{1+\varepsilon_{2} z}{1-\varepsilon_{2} z}
$$

where $\varepsilon_{1}=c-q e^{i \varphi} \sqrt{1-c^{2}}$ and $\varepsilon_{2}=c+q^{-1} e^{i \varphi} \sqrt{1-c^{2}}$ with $\varphi=\frac{1}{2} \arg v \in\left(-\frac{\pi}{2}, \frac{\pi}{2}\right]$, and $\lambda_{1}=1 /\left(1+q^{2}\right), \lambda_{2}=q^{2} /\left(1+q^{2}\right)$ and $q=\left(c \cos \varphi+\sqrt{1-c^{2} \sin ^{2} \varphi}\right) / \sqrt{1-c^{2}}$.

We remark that the above condition on $c_{1}=c$ and $c_{2}$ means that the equality $\left|c_{2}-c_{1}^{2}\right|=$ $1-\left|c_{1}\right|^{2}$ holds. Furthermore, the condition $c=1$ corresponds to the case when $\lambda_{1}=1$.

Note that $\left|\varepsilon_{1}\right|=\left|\varepsilon_{2}\right|=1$. Thus, they can be expressed by $\varepsilon_{1}=e^{i \theta_{1}}$ and $\varepsilon_{2}=e^{i \theta_{2}}$ for some $\theta_{1}, \theta_{2} \in \mathbb{R}$. Note also that

$$
\lambda_{1}=\frac{1-c^{2}}{2-c^{2}+c^{2} \cos 2 \varphi+c \sqrt{2(1+\cos 2 \varphi)-c^{2} \sin ^{2} 2 \varphi}} \quad \text { and } \quad \lambda_{2}=1-\lambda_{1} .
$$

It is convenient to notice the following fact. The function $f \in \mathcal{A}$ determined by the equation (2.1) for the function $p$ in the form of (2.2) should satisfy the equation

$$
\frac{f^{\prime}(z)}{f(z)}-\frac{1}{z}=\frac{1}{z}[\alpha+(1-\alpha) p(z)-1]=(1-\alpha)\left[\frac{2 \lambda_{1} \varepsilon_{1}}{1-\varepsilon_{1} z}+\frac{2 \lambda_{2} \varepsilon_{2}}{1-\varepsilon_{2} z}\right] .
$$

Integrating both sides, we obtain

$$
\log \frac{f(z)}{z}=-2(1-\alpha) \lambda_{1} \log \left(1-\varepsilon_{1} z\right)-2(1-\alpha) \lambda_{2} \log \left(1-\varepsilon_{2} z\right),
$$

and hence, the expression

$$
f(z)=\frac{z}{\left(1-\varepsilon_{1} z\right)^{2(1-\alpha) \lambda_{1}}\left(1-\varepsilon_{2} z\right)^{2(1-\alpha) \lambda_{2}}} .
$$

By taking the rotation $\varepsilon_{1} f\left(\overline{\varepsilon_{1}} z\right)=e^{i \theta_{1}} f\left(e^{-i \theta_{1}} z\right)$, we can replace $\varepsilon_{1}, \varepsilon_{2}$ by $1, \varepsilon=\overline{\varepsilon_{1}} \varepsilon_{2}=e^{i \theta_{0}}$, respectively, where $\theta_{0}=\theta_{2}-\theta_{1}$. Moreover, we may assume that $\operatorname{Im} \varepsilon \geq 0$ by considering $\overline{f(\bar{z})}$ if necessary. We now compute

$$
\begin{aligned}
\cos \theta_{0} & =\operatorname{Re}\left(\overline{\varepsilon_{1}} \varepsilon_{2}\right)=2 c^{2}-1-c \sqrt{1-c^{2}}\left(q-q^{-1}\right) \cos \varphi \\
& =2 c^{2} \sin ^{2} \varphi-1=c^{2}(1-\cos 2 \varphi)-1 .
\end{aligned}
$$

Thus an extremal function can be given in the form

$$
f(z)=\frac{z}{(1-z)^{2(1-\alpha) \lambda_{1}}\left(1-e^{i \theta_{0}} z\right)^{2(1-\alpha) \lambda_{2}}},
$$

where $\theta_{0}=\arccos \left[c^{2}(1-\cos 2 \varphi)-1\right]$.

For real constants $A, B, C$ we consider the quantity $\left|A+2 B e^{i \theta}+C e^{2 i \theta}\right|$ for $\theta \in \mathbb{R}$. Since this is invariant under the transformation $\theta \mapsto-\theta$, it is a function of $x=\cos \theta$. Thus we can define a function $\Phi:[-1,1] \rightarrow[0,+\infty)$ satisfying

$$
\Phi(\cos \theta)=\left|A+2 B e^{i \theta}+C e^{2 i \theta}\right| \text {. }
$$

We define $\operatorname{Sgn} x=x /|x|$ for $x \in \mathbb{R}$ with $x \neq 0$. The next elementary lemma (cf. $[3$, Lemma 3.3]) is useful in the sequel. 
Lemma 2.3. Let $A, B, C$ be real constants with $A C<0$ and set $\xi=-B(A+C) /(2 A C)$. Then the function $\Phi$ satisfies the inequality

$$
\Phi(x) \leq \begin{cases}\Phi(-1)=|A-2 B+C|=\operatorname{Sgn} B(-A+2 B-C) & \text { if } \xi \leq-1, \\ \Phi(\xi)=\sqrt{1-\frac{B^{2}}{A C}}|A-C| & \text { if }-1 \leq \xi \leq 1, \\ \Phi(1)=|A+2 B+C|=\operatorname{Sgn} B(A+2 B+C) & \text { if } 1 \leq \xi\end{cases}
$$

for $-1 \leq x \leq 1$.

\section{Proof of the Main theorem}

We are ready to show Theorem 1.1.

Let $f \in \mathcal{S}^{*}(\alpha)$ be of the form (1.1). Then we may write

$$
\frac{z f^{\prime}(z)}{f(z)}=\alpha+(1-\alpha) p(z)=1+2 \beta \sum_{n=1}^{\infty} c_{n} z^{n}
$$

for a Carathéodory function $p(z)=1+2\left(c_{1} z+c_{2} z^{2}+\cdots\right)$, where we put $\beta=1-\alpha$ for the sake of simplicity.

By a comparison of the coefficients, we have the relations

$$
\begin{aligned}
& a_{2}=2 \beta c_{1}, \\
& a_{3}=2 \beta^{2} c_{1}^{2}+\beta c_{2}, \\
& a_{4}=\frac{4}{3} \beta^{3} c_{1}^{3}+2 \beta^{2} c_{1} c_{2}+\frac{2}{3} \beta c_{3} .
\end{aligned}
$$

Let $g=f^{-1}$ be of the form (1.2). Then simple computations yield

$$
\begin{aligned}
& b_{2}=-a_{2}, \\
& b_{3}=-a_{3}+2 a_{2}, \\
& b_{4}=-a_{4}+5 a_{2} a_{3}-5 a_{2}^{3} .
\end{aligned}
$$

By using these relations, we have

$$
b_{4}=-\frac{2}{3} \beta\left(c_{3}-12 \beta c_{1} c_{2}+32 \beta^{2} c_{1}^{3}\right)=-\frac{2}{3} \beta Q,
$$

where $Q=c_{3}-12 \beta c_{1} c_{2}+32 \beta^{2} c_{1}^{3}$. Let $M=M(\beta)$ denote the quantity $\sup \left\{\left|c_{3}-12 \beta c_{1} c_{2}+32 \beta^{2} c_{1}^{3}\right|: p(z)=1+2 c_{1} z+2 c_{2} z^{2}+2 c_{3} z^{3}+O\left(z^{4}\right)\right.$ for some $\left.p \in \mathcal{P}\right\}$.

Thus we need to show

$$
M(\beta)= \begin{cases}\frac{(4 \beta-1)(8 \beta-1)}{\frac{2(3 \beta-1)^{3 / 2}(4 \beta-1)(8 \beta-1)}{\beta \sqrt{8 \beta-3}}} & \text { if } 1 \geq \beta>\beta_{1}=\frac{2}{5}, \\ \frac{1}{\beta}\left(\frac{12 \beta-1}{6}\right)^{3 / 2} & \text { if } \beta_{1} \geq \beta \geq \beta_{2}=\frac{13+\sqrt{33}}{48}, \\ 1 & \text { if } \beta_{2} \geq \beta \geq \beta_{3}=1-\alpha_{3}, \\ \text { if } \beta_{3} \geq \beta>0\end{cases}
$$


with enough information about the extremal functions, in addition. We now use Lemma 2.1 to parametrize the quantity $Q$ as

$$
\begin{aligned}
Q & =(8 \beta-1)(4 \beta-1) u^{3}-2(6 \beta-1)\left(1-|u|^{2}\right) u v-\left(1-|u|^{2}\right) \bar{u} v^{2}+\left(1-|u|^{2}\right)\left(1-|v|^{2}\right) w \\
& =A e^{3 i \psi}+2 B e^{i(\psi+\phi)}+C e^{i(2 \phi-\psi)}+\left(1-s^{2}\right)\left(1-t^{2}\right) w,
\end{aligned}
$$

where $u=s e^{i \psi}, v=t e^{i \phi}$ and

$$
\begin{aligned}
& A=(8 \beta-1)(4 \beta-1) s^{3}, \\
& B=-(6 \beta-1)\left(1-s^{2}\right) s t, \\
& C=-\left(1-s^{2}\right) s t^{2} .
\end{aligned}
$$

Note here that the condition $\left|c_{2}-c_{1}^{2}\right|=1-\left|c_{1}\right|^{2}$ is equivalent to $t=|v|=1$. Since the argument of $w$ can be taken arbitrarily, we have the sharp inequality

$$
\begin{aligned}
|Q| & \leq\left|A+2 B e^{i(\phi-2 \psi)}+C e^{2 i(\phi-2 \psi)}\right|+\left(1-s^{2}\right)\left(1-t^{2}\right) \\
& =\Phi(\cos (\phi-2 \psi))+\left(1-s^{2}\right)\left(1-t^{2}\right),
\end{aligned}
$$

where $\Phi$ is defined in (2.5). We next divide the proof into two cases.

Case I: When $0 \leq \alpha<\frac{3}{4}$, in this case $\frac{1}{4}<\beta \leq 1$.

We note that $8 \beta-1>6 \beta-1>4 \beta-1>0$. Hence $A>0, C<0$ and $B<0$ as long as st $>0$. In order to apply Lemma 2.3 , we compute

$$
\xi=-\frac{B(A+C)}{2 A C}=-\frac{(6 \beta-1)\left\{(8 \beta-1)(4 \beta-1) s^{2}-t^{2}\left(1-s^{2}\right)\right\}}{2 s^{2} t(4 \beta-1)(8 \beta-1)} .
$$

Therefore, by Lemma $2.3, M(\beta)$ is the supremum of the following quantity over $s, t \in$ $(0,1]$ :

$$
F(s, t)= \begin{cases}\Phi(-1)+\left(1-s^{2}\right)\left(1-t^{2}\right)=F_{1}(s, t) & \text { if } \xi \leq-1 \\ \Phi(\xi)+\left(1-s^{2}\right)\left(1-t^{2}\right)=F_{2}(s, t) & \text { if }-1 \leq \xi \leq 1 \\ \Phi(1)+\left(1-s^{2}\right)\left(1-t^{2}\right)=F_{3}(s, t) & \text { if } 1 \leq \xi\end{cases}
$$

Here,

$$
\begin{aligned}
& F_{1}(s, t)=(8 \beta-1)(4 \beta-1) s^{3}+2(6 \beta-1)\left(1-s^{2}\right) s t-\left(1-s^{2}\right) s t^{2}+\left(1-s^{2}\right)\left(1-t^{2}\right), \\
& F_{2}(s, t)=\sqrt{\frac{(6 \beta-1)^{2}-4 \beta^{2} s^{2}}{(8 \beta-1)(4 \beta-1)}}\left\{(8 \beta-1)(4 \beta-1) s^{2}+\left(1-s^{2}\right) t^{2}\right\}+\left(1-s^{2}\right)\left(1-t^{2}\right), \\
& F_{3}(s, t)=-(8 \beta-1)(4 \beta-1) s^{3}+2(6 \beta-1)\left(1-s^{2}\right) s t+\left(1-s^{2}\right) s t^{2}+\left(1-s^{2}\right)\left(1-t^{2}\right) .
\end{aligned}
$$

By its form, the function $F(s, t)$ is continuous on $[0,1]^{2}=[0,1] \times[0,1]$. We observe that $\xi \leq-1$ if and only if

$$
s^{2} \geq \frac{(6 \beta-1) t^{2}}{(6 \beta-1) t^{2}-2(8 \beta-1)(4 \beta-1) t+(8 \beta-1)(6 \beta-1)(4 \beta-1)}=\sigma_{2}(t)^{2},
$$

and that $\xi \geq 1$ if and only if

$$
s^{2} \leq \frac{(6 \beta-1) t^{2}}{(6 \beta-1) t^{2}+2(8 \beta-1)(4 \beta-1) t+(8 \beta-1)(6 \beta-1)(4 \beta-1)}=\sigma_{1}(t)^{2} .
$$




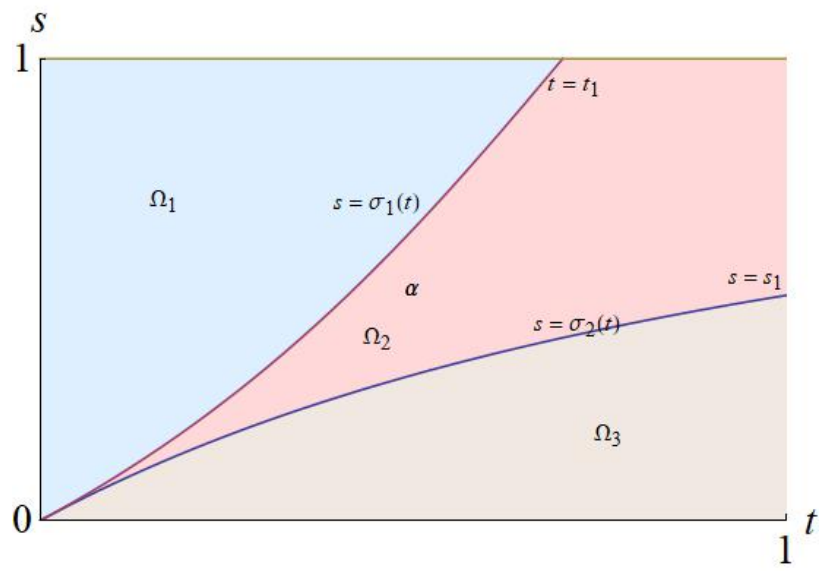

Figure 2. The regions $\Omega_{1}, \Omega_{2}, \Omega_{3}$ when $\frac{1}{4}<\beta<\frac{1}{2}$

Here, $0 \leq \sigma_{1}(t) \leq \sigma_{2}(t)$ and $\sigma_{1}(t)<1$ for $0 \leq t \leq 1$. Note that

$$
s_{1}:=\sigma_{1}(1)=\sqrt{\frac{6 \beta-1}{8 \beta^{2}(24 \beta-5)}} \in(0,1),
$$

which is a decreasing function in $\beta>0$. In view of the form of $1 / \sigma_{j}(t)^{2}$, we observe that $\sigma_{1}(t)$ and $\sigma_{2}(t)$ are monotone increasing and continuous on $0 \leq t \leq 1$ and $\sigma_{2}(t)=1$ when

$$
t=t_{1}:=\frac{6 \beta-1}{2} .
$$

For a fixed $\beta$, we divide the set $[0,1]^{2}$ into three closed regions $\Omega_{1}, \Omega_{2}, \Omega_{3}$ according as $\xi \leq-1,-1 \leq \xi \leq 1,1 \leq \xi$, respectively. More precisely, we can define them by $\Omega_{1}=\left\{(s, t) \in[0,1]^{2}: \sigma_{2}(t) \leq s\right\}, \Omega_{2}=\left\{(s, t) \in[0,1]^{2}: \sigma_{1}(t) \leq s \leq \sigma_{2}(t)\right\}, \Omega_{3}=$ $\left\{(s, t) \in[0,1]^{2}: s \leq \sigma_{1}(t)\right\}$.

Then

$$
M=M(\beta)=\max _{s, t \in[0,1]} F(s, t)=\max \left\{M_{1}, M_{2}, M_{3}\right\}
$$

where

$$
M_{j}=\max _{(s, t) \in \Omega_{j}} F_{j}(s, t), \quad j=1,2,3 .
$$

According to the conditions $t_{1} \in(0,1)$ and $t_{1} \geq 1$ of $t_{1}$, we consider two subcases to find the value $M(\beta)$.

Subcase Ia: When $\frac{1}{4}<\beta<\frac{1}{2}$, we have $t_{1} \in(0,1)$. See Figure 2 for the domains $\Omega_{1}, \Omega_{2}, \Omega_{3}$ in this subcase.

At this point, it is worth noting that $M \geq F_{j}(0,0)=1$ for any $\beta \in\left(\frac{1}{4}, \frac{1}{2}\right)$. Our strategy to compute $M$ is as follows. In order to find the value of $M_{j}$, we first look for critical points of the function $F_{j}$ in the interior Int $\Omega_{j}$ of $\Omega_{j}$. If there is no critical point, the maximum must be taken on the boundary $\partial \Omega_{j}$. Thus we have only to look at the boundary values of $F_{j}$. 
On the value $M_{1}$ : By the identity

$$
\frac{\partial F_{1}}{\partial t}(s, t)=2\left(1-s^{2}\right)\{(6 \beta-1+t) s-t\}
$$

critical points of $F_{1}$ in Int $\Omega_{1}$ lie on the curve $s=t /(6 \beta-1+t)$. Since

$$
\begin{aligned}
& \sigma_{2}(t)^{2}-\left(\frac{t}{6 \beta-1+t}\right)^{2} \\
= & \frac{4 \beta^{2} t^{2}(6 \beta-1-2 t)}{(6 \beta-1+t)^{2}\left\{(6 \beta-1) t^{2}-2(8 \beta-1)(4 \beta-1) t+(8 \beta-1)(6 \beta-1)(4 \beta-1)\right\}}>0
\end{aligned}
$$

for $0<t<t_{1}$, there is no critical point of $F_{1}(s, t)$ in Int $\Omega_{1}$. Thus the maximum $M_{1}$ of $F_{1}$ is taken on $\partial \Omega_{3}$. Simple computations yield

$$
\begin{aligned}
& F_{1}(1, t)=(8 \beta-1)(4 \beta-1), \\
& F_{1}(s, 0)=(8 \beta-1)(4 \beta-1) s^{3}+1-s^{2}=: \varphi(s) .
\end{aligned}
$$

Since $\varphi^{\prime}(s)=\{3(8 \beta-1)(4 \beta-1) s-2\} s$, the function $\varphi(s)$ is decreasing in $0<s<$ $2 /[3(8 \beta-1)(4 \beta-1)]$ and increasing in $2 /[3(8 \beta-1)(4 \beta-1)]<s$. In particular, the maximum of $F_{1}(s, 0)$ is taken at $s=0$ or $s=1$ on $[0,1]$. Noting that the function $F_{1}\left(\sigma_{2}(t), t\right)$ takes the value $F_{1}(0,0)=1$ for $t=0$ and $F_{1}\left(1, t_{1}\right)=F_{1}(1,0)$ for $t=t_{1}$, the maximum of $F_{1}$ on $\Omega_{1}$ is attained on the common boundary curve $s=\sigma_{2}(t)\left(0 \leq t \leq t_{1}\right)$ with $\Omega_{2}$. Hence, we conclude that $M_{1} \leq M_{2}$ so that $M=\max \left\{M_{2}, M_{3}\right\}$.

On the value $M_{2}$ : Since

$$
\frac{\partial F_{2}}{\partial t}=2 t\left(1-s^{2}\right)\left(\sqrt{1+\frac{4 \beta^{2}\left(1-s^{2}\right)}{(8 \beta-1)(4 \beta-1)}}-1\right)>0
$$

for $s, t \in(0,1)$, the function $F_{2}(s, t)$ is increasing in $0 \leq t \leq 1$ for a fixed $s \in(0,1)$. Therefore, the maximum $M_{2}$ is taken on either the segment $\left[s_{1}, 1\right] \times\{1\}$ or the common boundary $s=\sigma_{1}(t)(0 \leq t \leq 1)$ with $\Omega_{3}$. If we put

$$
M_{4}=\max _{s_{1} \leq s \leq 1} F_{2}(s, 1),
$$

then we have $M=\max \left\{M_{3}, M_{4}\right\}$.

We now look for the maximum of the function

$$
\eta(x)=(8 \beta-1)(4 \beta-1) F_{2}(\sqrt{x}, 1)^{2}=\left\{(6 \beta-1)^{2}-4 \beta^{2} x\right\}\left(32 \beta^{2} x-12 \beta x+1\right)^{2}
$$

on $s_{1}^{2} \leq x \leq 1$. Here we remark that $\max _{s_{1}^{2} \leq x \leq 1} \eta(x)=(8 \beta-1)(4 \beta-1) M_{4}^{2}$. Since

$$
\eta^{\prime}(x)=12 \beta\left(32 \beta^{2} x-12 \beta x+1\right)\left\{192 \beta^{3}-136 \beta^{2}+29 \beta-2-4 \beta^{2}(8 \beta-3) x\right\},
$$

the function $\eta(x)$ has possibly a critical point at

$$
x=\theta(\beta):=\frac{192 \beta^{3}-136 \beta^{2}+29 \beta-2}{4 \beta^{2}(8 \beta-3)} .
$$

In view of the formulae

$$
\theta(\beta)-1=\frac{(8 \beta-1)(4 \beta-1)(5 \beta-2)}{4 \beta^{2}(8 \beta-3)}
$$


and

$$
\theta(\beta)-s_{1}^{2}=\frac{(8 \beta-1)(4 \beta-1)\left(288 \beta^{2}-156 \beta+17\right)}{8 \beta^{2}(8 \beta-3)(24 \beta-5)},
$$

we see that $s_{1}^{2}<\theta(\beta)<1$ precisely when $(13+\sqrt{33}) / 48<\beta<2 / 5$ for $\beta$ under consideration. Note here that $(13+\sqrt{33}) / 48 \approx 0.390512>3 / 8$. Also, we note that $\eta^{\prime}(1)=12 \beta(8 \beta-1)^{2}(4 \beta-1)^{2}(5 \beta-2)$. According to the above observation, we consider the following three cases.

(i) When $1 / 4<\beta \leq(13+\sqrt{33}) / 48, \eta(x)$ is decreasing on $\left[s_{1}^{2}, 1\right]$ and therefore $F_{2}(s, 1)$ is decreasing on $\left[s_{1}, 1\right]$. Hence, we have $M_{4}=F_{2}\left(s_{1}, 1\right) \leq M_{3}$ because $\left(s_{1}, 1\right) \in \partial \Omega_{1}$.

(ii) When $(13+\sqrt{33}) / 48<\beta<2 / 5, \eta(x)$ is increasing on $\left[s_{1}^{2}, \theta(\beta)\right]$ and decreasing on $[\theta(\beta), 1]$. Hence $\eta(x)$ takes the maximum at $x=\theta(\beta)$. A straightforward calculation produces

and therefore

$$
\eta(\theta(\beta))=\frac{4(8 \beta-1)^{3}(4 \beta-1)^{3}(3 \beta-1)^{3}}{\beta^{2}(8 \beta-3)}
$$

$$
M_{4}=\frac{2(8 \beta-1)(4 \beta-1)(3 \beta-1)^{3 / 2}}{\beta(8 \beta-3)^{1 / 2}} .
$$

(iii) When $2 / 5 \leq \beta<1 / 2, \eta(x)$ is increasing on $\left[s_{1}^{2}, 1\right]$ and so is $F_{2}(s, 1)$ on $\left[s_{1}, 1\right]$. Thus $M_{4}=F_{2}(1,1)=(8 \beta-1)(4 \beta-1)$.

On the value $M_{3}$ : The last step is to solve the extremal problem on $\Omega_{3}$. To find a critical point of $F_{3}$, we solve the system of equations

$$
\begin{aligned}
& \frac{\partial F_{3}}{\partial t}=2\left(1-s^{2}\right)\{s(6 \beta-1+t)-t\}=0 \\
& \frac{\partial F_{3}}{\partial s}=-3(8 \beta-1+t)(4 \beta-1+t) s^{2}-2\left(1-t^{2}\right) s+t(12 \beta-2+t)=0 .
\end{aligned}
$$

Solving the first equation, we have the relation $s=t /(6 \beta-1+t)$. We substitute it into the second equation and simplify to

$$
\frac{12 \beta t\{(7 \beta-2) t+2(6 \beta-1)(3 \beta-1)\}}{(6 \beta-1+t)^{2}}=0 .
$$

Hence a candidate of critical points in Int $\Omega_{1}$ is only $\left(s_{0}, t_{0}\right)$, where

$$
s_{0}=\frac{2(1-3 \beta)}{\beta}, \quad t_{0}=-\frac{2(6 \beta-1)(3 \beta-1)}{7 \beta-2} .
$$

By the requirement $0<s_{0}<1$, the parameter $\beta$ should satisfy $2 / 7<\beta<1 / 3$. After some computations, we have the expression

$$
F_{3}\left(s_{0}, t_{0}\right)=145-\frac{16}{\beta}-432 \beta+432 \beta^{2}=: h(\beta) .
$$

Since $h^{\prime}(\beta)=16(6 \beta+1)(3 \beta-1)^{2} / \beta^{2}>0$ in this range, we have $F_{3}\left(s_{0}, t_{0}\right) \leq h(1 / 3)=$ $1=F_{3}(0,0)$. Therefore, this critical point does not contribute to the maximum of $F_{3}$. At any event, we conclude that the maximum $M_{3}$ is attained on the boundary of $\Omega_{3}$.

Next we shall look at the values of $F_{3}$ on $\partial \Omega_{3}$. First, $F_{3}(0, t)=1-t^{2} \leq 1=F(0,0)$ for $t \in[0,1]$. Second, we will prove the following result in the next section. 
Lemma 3.1. Let $1 / 4<\beta<1 / 2$. Then $F_{3}\left(\sigma_{1}(t), t\right) \leq \max \left\{1, F_{3}\left(s_{1}, 1\right)\right\}$ for $0 \leq t \leq 1$.

Therefore, we see that the maximum of $F_{3}$ on $\Omega_{3}$ is taken either on the segment $\left[0, s_{1}\right] \times$ $\{1\}$ or at the point $(0,0)$. We now have

$$
F_{3}(s, 1)=(12 \beta-1) s-32 \beta^{2} s^{3}=: G(s)
$$

for $s \in\left[0, s_{1}\right]$. Let

$$
s_{2}=\frac{\sqrt{12 \beta-1}}{4 \sqrt{6} \beta}
$$

which is the positive critical point of $G(s)$. Since

$$
s_{1}^{2}-s_{2}^{2}=-\frac{288 \beta^{2}-156 \beta+17}{96 \beta^{2}(24 \beta-5)},
$$

$s_{2} \in\left[0, s_{1}\right]$ if and only if $\beta \leq(13+\sqrt{33}) / 48$ for $\beta \in(1 / 4,1 / 2)$. So we divide the problem into two cases.

(1) When $1 / 4<\beta<(13+\sqrt{33}) / 48$, we have

$$
G(s) \leq G\left(s_{2}\right)=\frac{(12 \beta-1)^{3 / 2}}{6 \sqrt{6} \beta}
$$

for $0 \leq s \leq s_{1}$. Hence $M_{3}=\max \left\{1, G\left(s_{2}\right)\right\}$ in this case.

(2) When $(13+\sqrt{33}) / 48 \leq \beta<1 / 2, G(s)$ is increasing in $\left[0, s_{1}\right]$ so that

$$
G(s) \leq G\left(s_{1}\right)=\frac{9(8 \beta-1)(4 \beta-1) \sqrt{6 \beta-1}}{2 \sqrt{2} \beta(24 \beta-5)^{3 / 2}}
$$

for $s \in\left[0, s_{1}\right]$. Hence $M_{3}=\max \left\{1, G\left(s_{1}\right)\right\}$ in this case.

We now summarize what we have seen. So far, we had $M(\beta)=M=\max \left\{M_{3}, M_{4}\right\}$ and $M_{3}$ is computed in (1) and (2) above. On the other hand $M_{4}$ is computed in (i), (ii) and (iii) on the value $M_{2}$. Hence, we have the following conclusion.

(a) When $1 / 4<\beta \leq(13+\sqrt{33}) / 48=\beta_{2}$, we have $M=M_{3}=\max \left\{1, G\left(s_{2}\right)\right\}$. It is easy to see that $1 \leq G\left(s_{2}\right)=(12 \beta-1)^{3 / 2} / 6 \sqrt{6} \beta$ precisely if $\beta \geq \beta_{3}$, where $\beta_{3} \approx 0.31463$ is the unique real solution to the equation $(12 \beta-1)^{3}=6^{3} \beta^{2}$. That is,

$$
\beta_{3}=\frac{1}{24}(3+\sqrt[3]{13+2 \sqrt{11}}+\sqrt[3]{13-2 \sqrt{11}})
$$

which is the same as in (3.2).

Next we shall give extremal functions. First, for $1 / 4<\beta \leq \beta_{3}, M=1$ is attained when $s=0, t=0$ and $|w|=1$, which corresponds to $p(z)=(1+$ $\left.w z^{3}\right) /\left(1-w z^{3}\right)$. Taking $w=1$, we get $f=K_{\alpha, 3}$. Second, for $\beta_{3} \leq \beta \leq \beta_{2}$, $M=G\left(s_{2}\right)$ is attained when $t=1, s=s_{2}$ and $\cos \theta=1$, where $\theta=\phi-2 \psi$. We may assume $\psi=0$ so that $\theta=\phi=0$. Now we will apply Lemma 2.2 with $c=s_{2}, \varphi=\theta / 2=0$, where $s_{2}=\sqrt{\frac{11-12 \alpha}{4 \sqrt{6}(1-\alpha)}}$. Then $q=\left(1+s_{2}\right) / \sqrt{1-s_{2}^{2}}$ so that $\lambda_{1}=\left(1-s_{2}\right) / 2, \lambda_{2}=\left(1+s_{2}\right) / 2$ and $\varepsilon_{1}=-1, \varepsilon_{2}=-1$. Therefore, with the help of (2.4), we obtain the extremal function as in the third case of Theorem 1.1. 
(b) When $(13+\sqrt{33}) / 48<\beta<2 / 5$, we have $M=\max \left\{M_{3}, M_{4}\right\}$. Noting $M_{4} \geq 1$, we obtain, more precisely,

$$
M=\max \left\{\frac{9(8 \beta-1)(4 \beta-1) \sqrt{6 \beta-1}}{2 \sqrt{2} \beta(24 \beta-5)^{3 / 2}}, \frac{2(8 \beta-1)(4 \beta-1)(3 \beta-1)^{3 / 2}}{\beta(8 \beta-3)^{1 / 2}}\right\} .
$$

Letting $m_{3}$ be the first term in this maximum, we have

$$
\left(\frac{M_{4}}{m_{3}}\right)^{2}-1=\frac{\left(144 \beta^{2}-78 \beta+13\right)\left(288 \beta^{2}-156 \beta^{2}+17\right)^{2}}{81(6 \beta-1)(8 \beta-3)}>0
$$

for $\beta$ in the current range. Hence, we obtain

$$
M=M_{4}=\frac{2(8 \beta-1)(4 \beta-1)(3 \beta-1)^{3 / 2}}{\beta(8 \beta-3)^{1 / 2}} .
$$

Next let us see forms of extremal functions. Recall that $M_{4}$ was evaluated according as (i), (ii), (iii) occurs, respectively, in the analysis of the value $M_{2}$. First, for $\beta_{2}<\beta \leq \beta_{1}<2 / 5, M=M_{4}$ is attained when $s=\sqrt{\theta(\beta)}=: s_{3}, t=1$ and $\cos (\phi-2 \psi)=\xi$, where $\xi$ in (3.3) is now computed as

$$
\xi=-\frac{(6 \beta-1)\{(8 \beta-1)(4 \beta-1) \theta(\beta)-(1-\theta(\beta))\}}{2 \theta(\beta)(4 \beta-1)(8 \beta-1)} .
$$

We may take $\psi=0$ so that Lemma 2.2 is applicable with $\varphi=\phi / 2 \in(-\pi / 2, \pi / 2)$ and $c=s_{3}$. Now we obtain the form of an extremal function in the second case of Theorem 1.1 by (2.3) and (2.4), where we observe

$$
\cos \theta_{0}=s_{3}^{2}(1-\cos \phi)-1=\frac{4\left(72 \beta^{2}-41 \beta+5\right)}{8 \beta-3}=\frac{4\left(72 \alpha^{2}-103 \alpha+36\right)}{5-8 \alpha}
$$

and, after some simplifications,

$$
\lambda_{1}=\frac{(5 \beta-2)(8 \beta-1)}{144 \beta^{3}-58 \beta^{2}-2 \beta \sqrt{(3 \beta-1)(72 \beta-29)\left(24 \beta^{2}-15 \beta+2\right)}} .
$$

(c) When $2 / 5 \leq \beta<1 / 2$, similarly, we have

$$
M=\max \left\{\frac{9(8 \beta-1)(4 \beta-1) \sqrt{6 \beta-1}}{2 \sqrt{2} \beta(24 \beta-5)^{3 / 2}},(4 \beta-1)(8 \beta-1)\right\} .
$$

Let

$$
H(\beta)=\left(\frac{9 \sqrt{6 \beta-1}}{2 \sqrt{2} \beta(24 \beta-5)^{3 / 2}}\right)^{2}=\frac{81(6 \beta-1)}{8 \beta^{2}(24 \beta-5)^{3}} .
$$

Then

$$
H^{\prime}(\beta)=-\frac{81\left(288 \beta^{2}-75 \beta+5\right)}{4 \beta^{3}(24 \beta-5)^{4}}=-\frac{81\left\{2(12 \beta-2)^{2}+3(7 \beta-1)\right\}}{4 \beta^{3}(24 \beta-5)^{4}}<0
$$

for $2 / 5 \leq \beta<1 / 2$. Therefore, the function $H(\beta)$ is decreasing there so that

$$
H(\beta) \leq H(2 / 5)=\frac{354375}{389344}<1
$$

for $2 / 5 \leq \beta<1 / 2$. We thus get $M=(4 \beta-1)(8 \beta-1)$. 


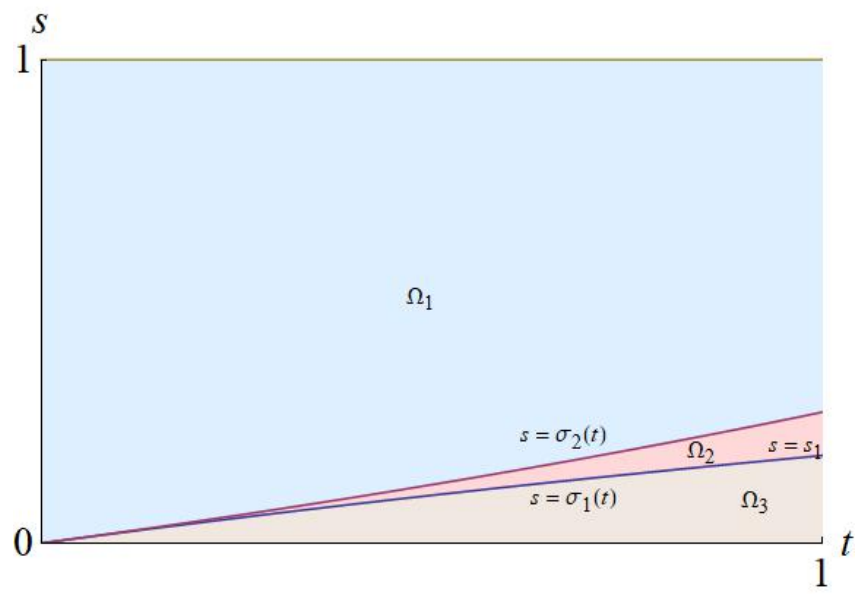

FiguRE 3 . The regions $\Omega_{1}, \Omega_{2}, \Omega_{3}$ when $\beta=1$

Since this case corresponds to the condition $s=|u|=1$, we have the simplest extremal function $f=K_{\alpha}$.

Subcase Ib: When $\frac{1}{2} \leq \beta \leq 1, t_{1} \geq 1$. We focus only on the special case $\beta=1$ since $\frac{1}{2} \leq \beta<1$ can be proved by the same technique showing in Case II. See Figure 3 for the domains $\Omega_{1}, \Omega_{2}, \Omega_{3}$ when $\beta=1$. Our aim is to show that $M(1)=21$.

If $\beta=1, F_{1}, F_{2}, F_{3}$ have the following forms.

$$
\begin{aligned}
& F_{1}(s, t)=21 s^{3}+10\left(1-s^{2}\right) s t-\left(1-s^{2}\right) s t^{2}+\left(1-s^{2}\right)\left(1-t^{2}\right), \\
& F_{2}(s, t)=\sqrt{\frac{25-4 s^{2}}{21}}\left[21 s^{2}+\left(1-s^{2}\right) t^{2}\right]+\left(1-s^{2}\right)\left(1-t^{2}\right), \\
& F_{3}(s, t)=-21 s^{3}+10\left(1-s^{2}\right) s t+\left(1-s^{2}\right) s t^{2}+\left(1-s^{2}\right)\left(1-t^{2}\right) .
\end{aligned}
$$

A computation shows that

$$
\begin{aligned}
\frac{\partial F_{1}}{\partial s} & =3 s^{2}\left(21-10 t+t^{2}\right)-2 s\left(1-t^{2}\right)+(10-t) t>0, \quad \text { for }(s, t) \in \Omega_{1} \\
\frac{\partial F_{2}}{\partial t} & =2 \sqrt{\frac{25-4 s^{2}}{21}}\left(1-s^{2}\right) t-2\left(1-s^{2}\right) t>0, \quad \text { for }(s, t) \in \Omega_{2} \\
F_{3}(s, t) & \leq-21 s^{3}+12<21, \quad \text { for }(s, t) \in \Omega_{3} .
\end{aligned}
$$

Therefore

$$
M(1)=\max _{s \in[0,1]}\left\{F_{1}(1, t), F_{2}(s, 1), 21\right\}=\max _{s \in[0,1]}\left\{\sqrt{\frac{25-4 s^{2}}{21}}\left(1+20 s^{2}\right), 21\right\}=21
$$

since $F_{1}(1, t) \equiv 21$ and $F_{2}\left(\sigma_{1}(t), t\right)=F_{3}\left(\sigma_{1}(t), t\right)<21$ for $t \in[0,1]$. The extremal function is the Koebe function $K_{0}$.

Case II: When $\frac{4}{3} \leq \alpha<1$, for any function $f(z)=z+\sum_{n=2}^{\infty} a_{n} z^{n} \in \mathcal{S}^{*}(\alpha)$, we have $f_{\gamma}(z)=\frac{1}{\gamma} f(\gamma z) \in \mathcal{S}^{*}(\alpha) \subset \mathcal{S}^{*}\left(\alpha^{\prime}\right)$ where $\alpha^{\prime}$ is any constant in $\left(\alpha_{3}, 3 / 4\right)$ and $\gamma^{3}=\frac{1-\alpha^{\prime}}{1-\alpha}>1$. 
In view of (3.1) and the result proved in Case I for $f_{\gamma}$, we have

$$
\left|\gamma^{3}\left(-a_{4}+5 a_{2} a_{3}-5 a_{2}^{3}\right)\right| \leq \frac{2}{3}\left(1-\alpha^{\prime}\right)
$$

which is

$$
\left|-a_{4}+5 a_{2} a_{3}-5 a_{2}^{3}\right| \leq \frac{2}{3}(1-\alpha) .
$$

On the other hand the function $K_{\alpha, 3}$ makes the equality hold.

In this way, we have proven (3.2) as required and finish the proof of the main theorem, up to Lemma 3.1.

\section{Proof of Lemma 3.1}

The remaining task is to show that $F_{3}\left(\sigma_{1}(t), t\right) \leq \max \left\{1, F_{3}\left(s_{1}, 1\right)\right\}$ for $0 \leq t \leq 1$, where $s_{1}=s_{1}(\beta)$ is given in (3.4). The standard method using Langrange multipliers seems to involve messy calculations. Thus we will take another approach. It is convenient in the sequel to consider the inverse function $\tau_{1}:\left[0, s_{1}\right] \rightarrow[0,1]$ of $\sigma_{1}:[0,1] \rightarrow\left[0, s_{1}\right]$. That is,

$$
\begin{aligned}
t=\tau_{1}(s) & =\frac{(8 \beta-1)(4 \beta-1) s^{2}+s \sqrt{(8 \beta-1)(4 \beta-1)\left\{(6 \beta-1)^{2}-4 \beta^{2} s^{2}\right\}}}{(6 \beta-1)\left(1-s^{2}\right)} \\
& =\frac{\kappa s^{2} /(6 \beta-1)+s \sqrt{\kappa\left(1-\mu s^{2}\right)}}{1-s^{2}}
\end{aligned}
$$

for $s \in\left[0, s_{1}\right]$, where

$$
\kappa=(8 \beta-1)(4 \beta-1)>0 \quad \text { and } \quad \mu=\frac{4 \beta^{2}}{(6 \beta-1)^{2}}=\frac{4 \beta^{2}}{\kappa+4 \beta^{2}} \in(0,1) .
$$

Then it is enough to show that the function

$$
w(s)=F_{3}\left(s, \tau_{1}(s)\right)
$$

satisfies $w(s) \leq \max \left\{1, F_{3}\left(s_{1}, 1\right)\right\}$ for $0 \leq s \leq s_{1}$. A straightforward computation gives the expression

where

$$
w(s)=1+\frac{s^{2}}{1+s} v(s),
$$

$$
v(s)=2 \kappa \mu s^{2}+(\kappa-1) s-(1+\kappa)+2 \kappa(1+\mu s) \sqrt{\frac{1-\mu s^{2}}{1-\mu}} .
$$

We now analyze the behaviour of $v(s)$ over $\left[0, s_{1}\right]$. First we compute

$$
v^{\prime}(s)=4 \kappa \mu s+\kappa-1+\frac{2 \kappa \mu\left(1-s-2 \mu s^{2}\right)}{\sqrt{(1-\mu)\left(1-\mu s^{2}\right)}} .
$$

We note here that $1-s-2 \mu s^{2} \geq 1-s_{1}-2 \mu s_{1}^{2}$ for $s \in\left[0, s_{1}\right]$. By using the expression

$$
1-s_{1}-2 \mu s_{1}^{2}=1-\frac{1}{2} \sqrt{\frac{6 \beta-1}{2 \beta(24 \beta-5)}}-\frac{1}{(6 \beta-1)(24 \beta-5)}=: u(\beta),
$$

we can check that $u^{\prime}(\beta)>0$ on $1 / 4<\beta<1 / 2$ and thus $u(\beta)$ is increasing there. We compute $u\left(\frac{17}{52}\right)=\frac{587}{925}-\frac{6}{17 \sqrt{37}}=0.0060 \cdots>0$. Hence, $1-s-2 \mu s^{2} \geq 0$ over $s \in\left[0, s_{1}\right]$ for 
$17 / 52 \leq \beta<1 / 2$. Note that $17 / 52 \approx 0.326923$. We divide the situation into four cases to show that $w(s) \leq \max \left\{1, w\left(s_{1}\right)\right\}$ over $\left[0, s_{1}\right]$.

Case I: When $\beta \in[3 / 8,1 / 2)$, we have $\kappa \geq 1$ and $1-s-2 \mu s^{2} \geq 1-s_{1}-2 \mu s_{1}^{2} \geq 0$ for $s \in\left[0, s_{1}\right]$. Then

$$
v^{\prime}(s) \geq 4 \kappa \mu s+\frac{2 \kappa \mu\left(1-s-2 \mu s^{2}\right)}{\sqrt{(1-\mu)\left(1-\mu s^{2}\right)}}>0
$$

for $s \in\left[0, s_{1}\right]$. Hence $w(s)$ is increasing in $\left[0, s_{1}\right]$ and therefore

$$
w(s) \leq w\left(s_{1}\right), \quad \text { for } s \in\left[0, s_{1}\right] .
$$

Case II: When $\beta \in\left[\frac{17}{52}, \frac{3}{8}\right]$, as we saw above, $\kappa \leq 1$. Since $\sqrt{1-\mu s_{1}^{2}} \leq \sqrt{1-\mu s^{2}} \leq 1$ for $0 \leq s \leq s_{1}$, we have

$$
\begin{aligned}
& \sqrt{1-\mu s^{2}} v^{\prime}(s) \\
= & (4 \kappa \mu s+\kappa-1) \sqrt{1-\mu s^{2}}+\frac{2 \kappa \mu\left(1-s-2 \mu s^{2}\right)}{\sqrt{1-\mu}} \\
\geq & 4 \kappa \mu s \sqrt{1-\mu s_{1}^{2}}+(\kappa-1)+\frac{2 \kappa \mu\left(1-s-2 \mu s^{2}\right)}{\sqrt{1-\mu}} \\
= & \frac{2 \kappa \mu}{\sqrt{1-\mu}}\left[-2 \mu s^{2}+\left(2 \sqrt{(1-\mu)\left(1-\mu s_{1}^{2}\right)}-1\right) s+1+\frac{(\kappa-1) \sqrt{1-\mu}}{2 \kappa \mu}\right] \\
= & \frac{2 \kappa \mu}{\sqrt{1-\mu}} f(s)
\end{aligned}
$$

for $s \in\left[0, s_{1}\right]$. Since $f(s)$ is concave, $f(s) \geq \min \left\{f(0), f\left(s_{1}\right)\right\}$ for $s \in\left[0, s_{1}\right]$. Noting the estimate $\sqrt{(4 \beta-1)(8 \beta-1)} \geq \frac{2 \sqrt{21}}{13}=0.7050 \cdots>7 / 10$, we obtain

$$
\begin{aligned}
f(0) & =1+\frac{(\kappa-1) \sqrt{(1-\mu)}}{2 \kappa \mu}=1-\frac{(3-8 \beta)(6 \beta-1)}{2 \beta \sqrt{(4 \beta-1)(8 \beta-1)}} \\
& \geq 1-\frac{5(3-8 \beta)(6 \beta-1)}{7 \beta}=\frac{3(16 \beta-5)(5 \beta-1)}{7 \beta}>0 .
\end{aligned}
$$

We also compute

$$
\begin{aligned}
f\left(s_{1}\right) & =1-2 \mu s_{1}^{2}+\frac{(\kappa-1) \sqrt{(1-\mu)}}{2 \kappa \mu}+\left(2 \sqrt{(1-\mu)\left(1-\mu s_{1}^{2}\right)}-1\right) s_{1} \\
& =1+\frac{3(4 \beta-1)(8 \beta-1)-2 \beta}{2 \beta(6 \beta-1)(24 \beta-5)}+\frac{(8 \beta-3)(6 \beta-1)}{2 \beta \sqrt{(4 \beta-1)(8 \beta-1)}}-\frac{1}{2 \beta} \sqrt{\frac{6 \beta-1}{48 \beta-10}} \\
& =: 1+q_{1}(\beta)+q_{2}(\beta)-q_{3}(\beta) .
\end{aligned}
$$

Simple calculations yield

$$
q_{1}^{\prime}(\beta)=\frac{-3\left(5-108 \beta+956 \beta^{2}-3648 \beta^{3}+4608 \beta^{4}\right)}{2 \beta^{2}(24 \beta-5)^{2}(6 \beta-1)^{2}}
$$


and

$$
q_{2}^{\prime}(\beta)=\frac{-3+54 \beta-300 \beta^{2}+544 \beta^{3}}{4 \beta[(4 \beta-1)(8 \beta-1)]^{3 / 2}} .
$$

One can easily check that the numerators of $q_{1}^{\prime}(\beta)$ and $q_{2}^{\prime}(\beta)$ are concave on the interval $\left[\frac{17}{52}, \frac{3}{8}\right]$, and that both of them take positive values at the end points $17 / 52$ and $3 / 8$. Therefore $q_{1}$ and $q_{2}$ are increasing on $\left[\frac{17}{52}, \frac{3}{8}\right]$. Since $q_{3}$ can be written in the form

$$
q_{3}(\beta)=\frac{1}{2 \beta} \sqrt{\frac{1}{8}+\frac{1}{4(48 \beta-10)}},
$$

it is obvious that it is decreasing on $\left[\frac{17}{52}, \frac{3}{8}\right]$. By virtue of the computation

$$
1+\left(q_{1}+q_{2}-q_{3}\right)\left(\frac{17}{52}\right)=\frac{23083}{15725}-\frac{125}{34 \sqrt{21}}-\frac{65}{17 \sqrt{37}}=0.037 \cdots>0,
$$

we conclude that $v^{\prime}(s)$ is positive on $\left[0, s_{1}\right]$. Therefore, no matter whether $v\left(s_{1}\right)$ is positive or negative, we have

$$
w(s)=1+\frac{s^{2}}{1+s} v(s) \leq \max \left\{w(0), w\left(s_{1}\right)\right\}=\max \left\{1, w\left(s_{1}\right)\right\}, \quad \text { for } s \in\left[0, s_{1}\right] .
$$

Case III: When $\beta \in[8 / 25,17 / 52]$, we observe that $\sqrt{1-\mu} \geq \sqrt{1-\mu(8 / 25)}=\sqrt{273} / 23=$ $0.71838 \cdots>51 / 71=1 / A$, because $\mu=\mu(\beta)$ is decreasing in $8 / 25 \leq \beta \leq 17 / 52$, where $A=71 / 51$. Note also $s_{1}(8 / 25)=\frac{25}{16} \sqrt{\frac{23}{134}}$. Then, for $s \in\left[0, s_{1}\right]$ we have

$$
\begin{aligned}
v(s) & =2 \kappa \mu s^{2}+(\kappa-1) s-(1+\kappa)+2 \kappa(1+\mu s) \sqrt{\frac{1-\mu s^{2}}{1-\mu}} \\
& \leq 2 \kappa \mu s^{2}+(\kappa-1) s-(1+\kappa)+2 A \kappa(1+\mu s)\left(1-\mu s^{2} / 2\right) \\
& =: g(s) .
\end{aligned}
$$

We will show that $g(s)$ in increasing on $\left[0, s_{1}\right]$ in this case. To do that, we compute

$$
\begin{aligned}
\frac{g^{\prime}(s)}{\kappa \mu} & =4 s+\frac{\kappa-1}{\kappa \mu}+A\left(2-2 s-3 \mu s^{2}\right) \\
& =2(2-A) s+\frac{\kappa-1}{\kappa \mu}+A\left(2-3 \mu s^{2}\right),
\end{aligned}
$$

which is a concave function of $s$. Thus $g^{\prime}(s) \geq \min \left\{g^{\prime}(0), g^{\prime}\left(s_{1}\right)\right\}$ for $s \in\left[0, s_{1}\right]$. Introducing the new variable $x=16 \beta-3$, we compute

$$
\frac{\kappa-1}{\kappa \mu}=\frac{(6 \beta-1)^{2}(8 \beta-3)}{\beta(4 \beta-1)(8 \beta-1)}=\frac{(x-3)(3 x+1)^{2}}{\left(x^{2}-1\right)(x+3)}=: h(x) .
$$

Note that the range of $x$ is now $\left[\frac{53}{25}, \frac{29}{13}\right]$. Since

$$
h^{\prime}(x)=\frac{16\left(x^{3}+3\right)(3 x+1)}{\left(x^{2}-1\right)^{2}(x+3)^{2}}>0,
$$


THE INVERSE OF A STARLIKE FUNCTION OF POSITIVE ORDER

for $x \in\left[\frac{53}{25}, \frac{29}{13}\right], h(x)$ is increasing and so that $g^{\prime}(0) /(\kappa \mu)=h(x)+2 A \geq h\left(\frac{53}{25}\right)+2 A=$ $\frac{46}{\sqrt{273}}-\frac{5819}{2184}=0.1196 \cdots>0$. We also observe that $h(x)$ is convex on $1<x<+\infty$ by

$$
h^{\prime \prime}(x)=-\frac{16\left(6 x^{6}+3 x^{5}+9 x^{4}+82 x^{3}+108 x^{2}+27 x+21\right)}{(x-1)^{3}(x+1)^{3}(x+3)^{3}} .
$$

In particular, $(\kappa-1) /(\kappa \mu)$ is a concave function of $\beta$ on $(1 / 4,1 / 2)$. Secondly, another computation yields

$$
\frac{g^{\prime}\left(s_{1}\right)}{\kappa \mu}=2(2-A) s_{1}(\beta)+\frac{\kappa-1}{\kappa \mu}+\frac{A}{2} \cdot \frac{576 \beta^{2}-216 \beta+17}{(6 \beta-1)(24 \beta-5)} .
$$

On the interval $\left[\frac{8}{25}, \frac{17}{52}\right]$, we estimate, by convexity,

$$
2(2-A) s_{1}^{\prime}(\beta)>2 s_{1}^{\prime}(1 / 4)=-20
$$

and, by concavity,

$$
\frac{d}{d \beta}\left(\frac{\kappa-1}{\kappa \mu}\right)=16 h^{\prime}(16 \beta-3) \geq 16 h^{\prime}(7 / 3)=\frac{1431}{50}>20 .
$$

Hence the sum of the first two terms of $g^{\prime}\left(s_{1}\right) /(\kappa \mu)$ in $(4.1)$ is an increasing function of $\beta$ on $[8 / 25,17 / 52]$. On the other hand, by using the same change of variables as before, we have

$$
\frac{576 \beta^{2}-216 \beta+17}{(6 \beta-1)(24 \beta-5)}=\frac{2\left(9 x^{2}-13\right)}{(3 x-1)(3 x+1)}=2-\frac{24}{9 x^{2}-1}
$$

which is obviously increasing in $x>1 / 3$. Therefore, the third term is also increasing in $\beta$. In summary, $g^{\prime}\left(s_{1}\right) /(\kappa \mu)$ is increasing in $\beta$ so that its minimum over $\left[\frac{8}{25}, \frac{17}{52}\right]$ is taken at $\beta=8 / 25$ and its value is

$$
\frac{g^{\prime}\left(s_{1}(8 / 25)\right)}{\kappa \mu}=\frac{34312 \sqrt{273}+13650 \sqrt{3082}-575 \sqrt{841386}-779746}{292656}=0.0599 \cdots>0 .
$$

Hence we have proved that $g(s)$ is increasing on $\left[0, s_{1}\right]$ for each $\beta \in\left[\frac{8}{25}, \frac{17}{52}\right]$. Thus we need only to show that the value $g\left(s_{1}\right)$ is negative. Now we write $g\left(s_{1}\right)$ in the form

$$
\begin{aligned}
g\left(s_{1}\right) & =2 \kappa \mu s_{1}^{2}+(\kappa-1) s_{1}-(1+\kappa)+2 A \kappa\left(1+\mu s_{1}\right)\left(1-\mu s_{1}^{2} / 2\right) \\
& =(2-A)\left[\kappa \mu s_{1}^{2}\right]+\left[(\kappa-1) s_{1}\right]+[2 A \kappa-\kappa-1]+2 A\left[\mu \kappa s_{1}\right]+A\left[-\kappa \mu^{2} s_{1}^{3}\right] \\
& =(2-A) T_{1}+T_{2}+T_{3}+2 A T_{4}+A T_{5} .
\end{aligned}
$$


By virtue of

$$
\begin{aligned}
T_{1}^{\prime}(\beta) & =\frac{16 \beta-3}{(24 \beta-5)^{2}(6 \beta-1)^{2}}, \\
T_{2}^{\prime}(\beta) & =\frac{1152 \beta^{2}-456 \beta+49}{(24 \beta-5)^{2}} \sqrt{\frac{48 \beta-10}{6 \beta-1}}, \\
T_{3}(\beta) & =(2 A-1)(4 \beta-1)(8 \beta-1)-1, \\
T_{4}(\beta) & =\sqrt{2} \beta \sqrt{\frac{(4 \beta-1)(8 \beta-1)}{(6 \beta-1)^{2}}} \sqrt{2 T_{1}(\beta),} \\
T_{5}(\beta) & =-\frac{1}{\sqrt{(48 \beta-10)(6 \beta-1)}} \frac{(4 \beta-1)(8 \beta-1) \beta}{(6 \beta-1)^{3}(24 \beta-5)} \\
& =:-\frac{1}{\sqrt{(48 \beta-10)(6 \beta-1)}} t_{5}(\beta)
\end{aligned}
$$

and

$$
t_{5}^{\prime}(\beta)=\frac{5-60 \beta-24 \beta^{2}+1920 \beta^{2}-4608 \beta^{4}}{(24 \beta-5)^{2}(6 \beta-1)^{4}}<0, \quad \text { for } \beta \in\left[\frac{8}{25}, \frac{17}{52}\right]
$$

by the concavity of the numerator of $t_{5}^{\prime}(\beta)$, each $T_{j}=T_{j}(\beta)$ is increasing in $8 / 25 \leq \beta \leq$ $17 / 52$. We thus obtain

$$
g\left(s_{1}\right) \leq g\left(s_{1}(17 / 52)\right)=\frac{7670451 \sqrt{37}-54764625}{945465625}=-0.00857 \cdots<0 .
$$

Hence, we see that $v(s)$ is always negative on $\left[0, s_{1}\right]$ for $\beta \in\left[\frac{8}{25}, \frac{17}{52}\right]$. Therefore in this case

$$
w(s) \leq 1, \quad \text { for } s \in\left[0, s_{1}\right] .
$$

Case IV: When $\beta \in\left[\frac{1}{4}, \frac{8}{25}\right]$, we have the estimates

$$
\begin{aligned}
v(s) & =2 \kappa \mu s^{2}+(\kappa-1) s-(1+\kappa)+2 \kappa(1+\mu s) \sqrt{\frac{1-\mu s^{2}}{1-\mu}} \\
& \leq 2 \kappa \mu s^{2}+(\kappa-1) s-(1+\kappa)+2 A \kappa(1+\mu s) \\
& =: l(s) \\
& \leq \max \left\{l(0), l\left(s_{1}\right)\right\}
\end{aligned}
$$

for $s \in\left[0, s_{1}\right]$, where $A=71 / 51$ as before. Next we will show that both $l(0)$ and $l\left(s_{1}\right)$ are negative. First, since $A<7 / 5$, we have

$$
l(0)=-(1+\kappa)+2 A \kappa<-1-\kappa+\frac{14}{5} \kappa=\frac{4}{5}(3 \beta-1)(24 \beta-1)<0 .
$$

Second, a computation shows that

$$
\begin{aligned}
l\left(s_{1}\right) & =2 \kappa \mu s_{1}^{2}+(\kappa-1) s_{1}-(1+\kappa)+2 A \kappa\left(1+\mu s_{1}\right) \\
& =2\left[\kappa \mu s_{1}^{2}\right]+\left[(\kappa-1) s_{1}\right]+[2 A \kappa-\kappa-1]+2 A\left[\mu \kappa s_{1}\right] \\
& =2 T_{1}+T_{2}+T_{3}+2 A T_{4},
\end{aligned}
$$


where $T_{1}, T_{2}, T_{3}$ and $T_{4}$ have the same form as in Case III. The same arguments as in Case III show that all the terms $T_{j}=T_{j}(\beta)$ are increasing in $\beta$ as before. Hence, we obtain

$$
l\left(s_{1}\right) \leq l\left(s_{1}(8 / 25)\right)=\frac{37025 \sqrt{3082}-4054049}{110759375}=-0.0180 \cdots<0 .
$$

We find that $v(s)$ is always negative on $\left[0, s_{1}\right]$ for $\beta \in\left[\frac{1}{4}, \frac{8}{25}\right]$. Therefore in this case too, we have

$$
w(s) \leq 1, \quad \text { for } s \in\left[0, s_{1}\right] .
$$

Considering the above four cases together, we finally proved that $w(s) \leq \max \left\{1, w\left(s_{1}\right)\right\}$ over $\left[0, s_{1}\right]$.

\section{REFERENCES}

1. M. F. Ali and A. Vasudevarao, Coefficient estimates of negative powers and inverse coefficients for certain starlike functions, Proc. Indian Acad. Sci. Math. Sci. 127 (2017), 449-462.

2. J. T. P. Campschroer, Inverse coefficients and symmetrzation of univalent functions, Thesis of Catholic University, Nijmegen (1984).

3. J. H. Choi, Y. C. Kim, and T. Sugawa, A general approach to the Fekete-Szegö problem, J. Math. Soc. Japan 59 (2007), 707-727.

4. W. K. Hayman, Multivalent Functions, Second edition, Cambridge University Press, London, 1994.

5. G. P. Kapoor and A. K. Mishra, Coefficient estimates for inverses of starlike functions of positive order, J. Math. Anal. Appl. 329 (2007), 922-934.

6. J. G. Krzyż, R. J. Libera, and E. Złotkiewicz, Coefficients of inverse of regular starlike functions, Ann. Univ. Mariae Curie-Skłodowska. Sect. A 33 (1979), 103-109.

7. M. Li, A note on successive coefficients of spirallike functions, Filomat 32 (2018), 1199-1207.

8. M. Li and T. Sugawa, Schur parameters and Carathéodory class, Result Math. 74 (2019), \# 185.

9. R. J. Libera and E. J. Złotkiewicz, Early coefficients of the inverse of a regular convex function, Proc. Amer. Math. Soc. 85 (1982), 225-230.

10. K. Löwner, Untersuchungen über schlichte konforme Abbildungen des Einheitskreises. I, Math. Ann. 89 (1923), 103-121.

11. D. V. Prokhorov and J. Szynal, Inverse coefficients for $(\alpha, \beta)$-convex functions, Ann. Univ. Mariae Curie-Skłodowska. Sect. A 35 (1981), 125-143.

12. M. S. Robertson, On the theory of univalent functions, Ann. of Math. 37 (1936), 374-408.

Graduate School of Information Sciences, Tohoku University, Aoba-ku, Sendai 9808579 , JAPAN

E-mail address: sugawa@math.is.tohoku.ac.jp

School of Statistics, University of International Business and Economics, No. 10, Huixin Donguie, Chaoyang District, Beijing 100029, China

E-mail address: wangmabel@163.com 